Kopp, O.; Markert, Steffen; Tornow, Ralf Peter:

Comparing light sensitivity, linearity and step response of electronic cameras for ophthalmology

\footnotetext{
Zuerst erschienen in: $\quad$ Biomedizinische Technik = Biomedical Engineering. - Berlin [u.a.] : de Gruyter. - 47 (2002), S1b, S. 602-605.

Jahrestagung der Deutschen Gesellschaft für Biomedizinische Technik (DGBM) im VDE ; 36 (Karlsruhe) : 2002.09.25-27

Erstveröffentlichung: 2002

Datum Digitalisierung: $\quad 2009-11-26$

ISSN (online): $\quad$ 1862-278X

ISSN(print) 0013-5585

DOI: $\quad$ 10.1515/bmte.2002.47.s1b.602

[Zuletzt gesehen: 2019-12-06]
}

„Im Rahmen der hochschulweiten Open-Access-Strategie für die Zweitveröffentlichung identifiziert durch die Universitätsbibliothek IImenau."

"Within the academic Open Access Strategy identified for deposition by IImenau University Library."

„Dieser Beitrag ist mit Zustimmung des Rechteinhabers aufgrund einer (DFGgeförderten) Allianz- bzw. Nationallizenz frei zugänglich."

„This publication is with permission of the rights owner freely accessible due to an Alliance licence and a national licence (funded by the DFG, German

Research Foundation) respectively."

\section{DFG}

Nationallizenzen 


\title{
COMPARING LIGHT SENSITIVITY, LINEARITY AND STEP RESPONSE OF ELECTRONIC CAMERAS FOR OPHTHALMOLOGY
}

\author{
O. Kopp, S. Markert, R.P. Tornow \\ Institut für Biomedizinische Technik, Technische Universität Ilmenau, Deutschland \\ ralf.tornow@informatik.tu-ilmenau.de
}

\begin{abstract}
Purpose: To develop and test a procedure to measure and compare light sensitivity, linearity and step response of electronic cameras. Methods: The pixel value (PV) of digitized images as a function of light intensity (I) was measured. The sensitivity was calculated from the slope of the $P(I)$ function, the linearity was estimated from the correlation coefficient of this function. To measure the step response, a short sequence of images was acquired. During acquisition, a light source was switched on and off using a fast shutter. The resulting $P V$ was calculated for each video field of the sequence. Results: $A C C D$ camera optimized for the near-infrared (IR) spectrum showed the highest sensitivity for both, visible and IR light. There are little differences in linearity. The step response depends on the procedure of integration and read out.
\end{abstract}

\section{Keywords - CCD camera, CMOS camera, Imaging, Image sensor, Ophthalmology}

\section{Introduction}

Direct digital acquisition of images using an electronic camera offers several advantages over conventional photography. These images can easily be analyzed, enhanced, archived and transferred electronically. For instance, compressed digital retinal images could be used for telemedicine [1]. Recently, a method was described to calculate the macular pigment distribution in children from digitized fundus images [3]. In both cases conventional fundus photographs were scanned to produce digitized images. This step could be omitted by direct digital acquisition. The resolution of several digital color cameras was measured and compared to film material. High end digital cameras match the film resolution closely [2]. However, these high resolution cameras need a long time for image data transfer (up to $25 \mathrm{~s}$ [2]). Besides resolution, other important parameters of electronic cameras are light sensitivity, linearity and the response to fast changes in light intensity (step response). Linearity is a stringent requirement for quantitative imaging. The camera must be linear in order to perform image analysis such as arithmetic ratios, shading correction, linear transformations, etc. More than this, any offset has to be compensated.

For special applications, a procedure was developed to measure and compare light sensitivity, linearity, offset and step response of cameras that allow imaging at video rate. This procedure was applied to measure canneras that are available at present and it will be useful in future to evaluate the improvements of new cameras.

\section{Materials}

Cameras with the following image sensors were tested: $b / w-C C D$ for the visible range (vis-CCD), b/w-CCD for the near infrared range (IR-CCD), 3-chip-color-CCD (3chip-CCD), b/w-CMOS (CMOS). All CCD cameras used CCIR video standard (interlaced), the CMOS camera was equipped with IEEE 1394 interface. In interlaced video mode, the entire video frame is divided into two fields, the odd field that contains all odd video lines $(1,3,5$ to 493) and the even field, that contains all even video lines $(2,4,6$ to 494). The 3-chip-CCD could be set to either field or frame integration mode. Field and frame integration mode differ mainly in the timing of charge integration and read out. In field integration mode, the integration time is $20 \mathrm{~ms}$ for every field and there is no overlap in the integration time of the two fields. In frame integration mode, the integration time for every field is $40 \mathrm{~ms}$. This results in an overlap of the integration time of $20 \mathrm{~ms}$ for the two fields. Consequently, when using continuous lighting, the effective exposure time for one frame is $60 \mathrm{~ms}$. Additionally, the vertical resolution is different among the two modes. For details see e.g. [4,5]. The 3-chip-CCD and the vis-CCD were optimized for the visible range and therefore they are equipped with IR blocking filters.

A CMOS camera was included in the measurements to compare its sensitivity with the CCD cameras. The settings of the CMOS camera (pixel number of the actice area, integration time, frame rate etc.) could be changed in a large range. For the comparison with the CCIR video cameras, the parameters were set close to the values of the CCD cameras: resolution $768 \times 512$ pixels, integration time $40 \mathrm{~ms}$. However, in this mode, the frame rate of the CMOS camera is restricted to 11 frames per second $(f / s)$. To get a frame rate of $25 \mathrm{f} / \mathrm{s}$, the resolution has to be reduced to $768 \times 256$ pixels.

Table 1: cameras / image sensors

\begin{tabular}{lcl}
\hline camera & number of pixels & image transfer \\
\hline vis-CCD & $768 \times 494$ & CCIR video \\
IR-CCD & $768 \times 494$ & CCIR video \\
3-chip-CCD & $752 \times 582$ & CCIR videw \\
CMOS, set to & $768 \times 512$ & IFEl 1394 \\
\hline
\end{tabular}

\section{Methods}

The experimental set up is shown in fig. 1. Two difleren light sources were used. For the visihle rampec, a cold light source with IR filter (Volpi) was used. The infenst! of llus light source could be tuncel without changeng the yeichal 


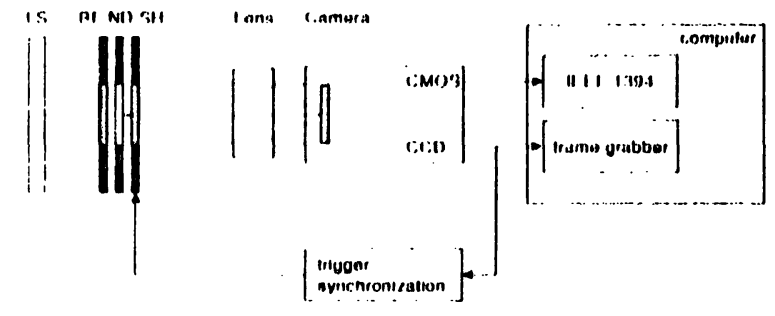

Higure 1: fixperimental set-up). LS: light source, BF: bandpass lilter, NI): neutral density filter, $\mathrm{SH}$ : shutter.

distribution. The light intensity was measured using a phowometer (Minolta). For the IR range, a tungsten bulb wiss used. In this case, neutral density (ND) filters were usced to change the light intensity. The ND-filters were callibrated for the measured spectral range. For both, the visible and the IR range, the spectral distribution of the light was restricted by bandpass filters with full width at half maximum (FWHM) of about $25 \mathrm{~nm}$ and central wavelength of $555 \mathrm{~nm}$ and 85() $\mathrm{nm}$, respectively.

Shutter: A ferro-electric liquid crystal shutter with rise and fall time of less than $50 \mu$ s and a contrast ratio of 100:1 was used. The synchronization of the shutter with the video signal was realized using a circuit similar to that described in [6]. This circuit allows to trigger the shutter exactly during the vertical blanking period between two video frames.

I.ens: Schneider Xenoplan 1.4/23, C-mount, set to F 5.6 Image acquisition: For the $\mathrm{CCD}$ cameras, an imaging board (FlashBus MVlite, Integral Technologies) and Image-Pro-Plus software (Media cybernetics) were used for image acquisition. Sequences of 50 videoframes could be digitized in real-time. For the CMOS camera, a purpose written software was used for image acquisition. The parameters for image acquisition (active area, exposure time, frame rate etc.) could be pre-selected.

Image analysis: The image analysis was performed using Image-Pro-Plus software. In each image, the average pixel value was calculated in an area of interest (AOI) of $100 \times 100$ pixels.

Sensitivity: The data points (pixel value $P$ as a function of light intensity $I$ ) were fitted to a linear function

$$
\mathrm{P}(I)=a^{*} I+b
$$

There are different definitions of "sensitivity" for electronic cameras. Here, the sensitivity $S$ is defined as the slope $a$ of the linear function $\mathrm{P}(I)$. In this way, the sensitivity $S$ is independent of the offset $b$ (see below). Here, only the relative sensitivity of the different cameras was evaluated, however, the procedure described here allows to measure absolute sensitivity.

Linearity: There is no standard method for measuring linearity. Here, the correlation coefficient $r^{2}$ of the function $\mathrm{P}(I)$ was used as a measure of linearity.

Offset: An offset could be introduced by either the camera or the imaging board. For quantitative imaging, the offset has to be measured and compensated. The procedure described here has the advantage that it measures the

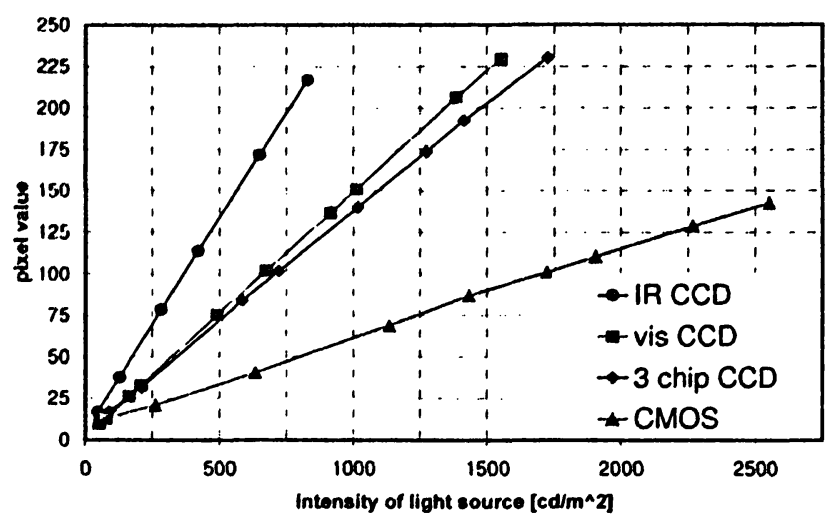

Figure 2: Pixel value as a function of light intensity

resulting offset of the entire chain from the light intensity to the resulting pixel value of the digitized image.

Step response: A short image sequence was acquired. During the image acquisition, the shutter was switched on for a short time, e.g. two video frames $(80 \mathrm{~ms})$. The transition (on or off) was triggered exactly during the vertical blanking period between two video frames. A purpose written software was used to separate the two fields of a video frame and to calculate the pixel value of the odd and even fields of the acquired frames separately. For all measurements, automatic gain control was switched off and gamma set to 1 . The step response was not measured for the CMOS camera.

\section{Results}

Sensitivity: The pixel values of the digitized images as a function of light intensity for green light for all tested cameras are shown in fig. 2. For the 3-chip-CCD camera, only the green channel was measured, because the two other channels (blue and red) are insensitive to green light. All cameras show a linear behavior with different slopes and offset. Fig. 3 shows the relative sensitivity of the cameras for the green (fig. 3a) and the IR (fig. 3b) range. For green light, the IR-CCD has the highest sensitivity. The two CCD cameras optimized for the visible spectrum show only about half the sensitivity of the IR-CCD and the CMOS camera has the lowest sensitivity in the visible spectrum. Fig. $3 b$ shows the relative sensitivity for the IR range. These values were calculated from the $P(I)$-function measured in the IR range similar to that shown in fig. 2 . For the IR range, the IR-CCD has the highest sensitivity and the CMOS camera has less than $40 \%$ of this sensitivity. Without modifications, the two CCD cameras optimized for the visible spectrum are insensitive to $\mathbb{R}$ light. The result for the vis-CCD shown in fig. $3 \mathrm{~b}$ was measured without IR filter. The IR filter of the 3-chip-CCD camera could not be removed and therefore, all channels are insensitive for IR light.

Linearity and ofiset: The correlation coefficient $r^{2}$ and the offset for all cameras (measured using green light) are shown in table 2. Compared to the CMOS camera, the CCD cameras have better linearity and lower offset. 

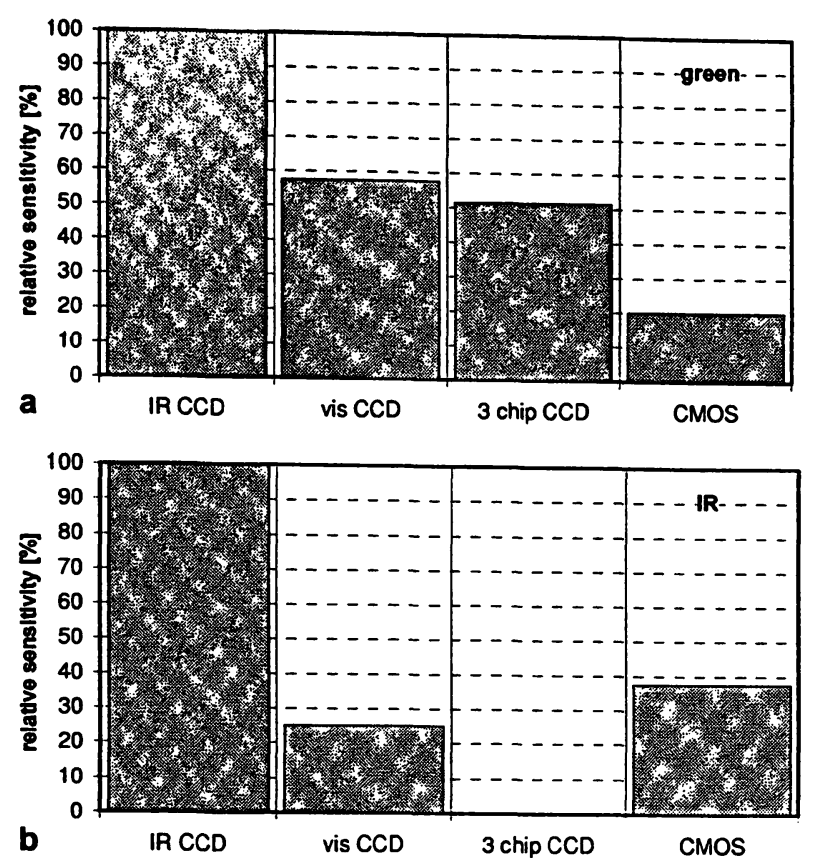

Figure 3: Relative sensitivity for green (a) and IR light (b)

Table 2: linearity and offset

\begin{tabular}{lcc}
\hline camera / sensor & $\mathbf{r}^{\mathbf{2}}$ & offset [PV] \\
\hline vis-CCD & 0,9999 & 2,1 \\
IR-CCD & 0,9999 & 5,5 \\
3-chip-CCD & 0,9997 & 5,5 \\
CMOS & 0,9991 & 10,3 \\
\hline
\end{tabular}

Step response: Fig. 4 shows the pixel values in each field for all tested cameras (a-d) and the time course of the light intensity (e). The time course of the light intensity shown in fig. $4 \mathrm{e}$ corresponds to the integration time of the camera. The corresponding video signal is $20 \mathrm{~ms}$ delayed. The 3chip-CCD camera in field integration mode (Fig. 4d) shows a fast response, the intensity of each video field follows the intensity of the light source. On the other hand, for the 3-chip-CCD camera in frame integration mode and the two other cameras, the first field after the light is switched on shows only approximately half the pixel value compared to the following fields. Hence, the average pixel value of the first frame (both fields together) is about 75\% of the following frames. When the light is switched off, a similar effect can be seen.

\section{Discussion}

Sensitivity: There are large differences in sensitivity among cameras for both, the visible and IR spectrum. One reason, among others, might be the use of image sensors with different technology. Many parameters like sensitivity, dynamic range and signal to noise ratio have been greatly improved during the last years. However, the technology of the image sensor of a given camera is not always known. To get the most sensitive camera for a special application, the sensitivity should be measured under real experimental conditions, including light Icvel, spectral range and exposure time.
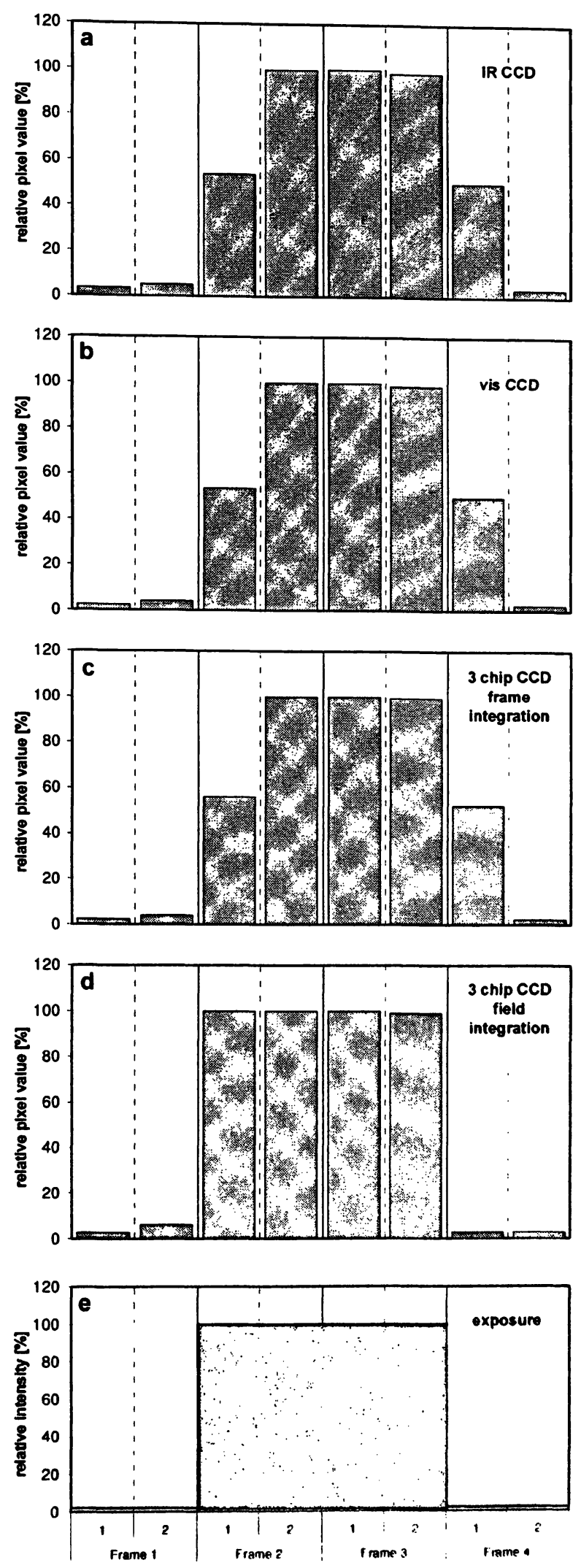

Figure 4: Step response

Linearity: There are only small differences in lincinnt! between the CCD cimneras. The smalles value for the CMOS camera might be due to the fact that the intiom.t finer of the computer was uned for integhatenen time 
comtrol. This timer has an accuracy of about $\pm 1 \mathrm{~ms}$ resulting in an error for the exposure time (4t) ms) of about $2.5 \%$.

()frset: laor quantitative imaging. the offiset has to be mleisured and compensalled. Many imaging boards allow (1) adjust the reference levels of the input $\Lambda D$-converter so Ihat an offiset call be compensated during image acyuisition. If this is not the calse, the stored images have of be compensilted for the offiset. The offset of the ( MIOS camera can not be compensated during image alcyuisition and so this has to be done afterwards.

sicp response: The step response shows important diflerences among the cameras due to the different prociedure of integration and read out. In field integration mode, the integration time is restricted to $20 \mathrm{~ms}$. For cxilmple, field 1 in frame 2 (see fig. 4d) is exposed only during these $20 \mathrm{~ms}$. However, in frame integration mode, the integration time is $40 \mathrm{~ms}$. Field 1 in frame 2 (fig. $4 \mathrm{a}-\mathrm{c}$ ) is exposed during field 2 of frame 1 and field 1 of frame 2 . $\Lambda s$ in this case, the shutter is switched on after half the integration time, this field shows only half the intensity of the following field. The following field (field 2 of frame 2) is exposed during field 1 and 2 of frame 2 and thus shows full intensity. A similar behavior can be seen in field 1 of frame 4 when the light is switched off.

\section{Conclusion}

There are a number of different cameras and image sensors. Due to the fast development in this field, the number is still increasing. However, there is no ideal image sensor that fulfils all needs like high resolution, high frame rate, high sensitivity, low noise etc. Instead, the optimal camera / image sensor depends on the special application and has to be carefully selected. For a given application, it is important to be able to measure and compare selected features of different cameras fast and reliably, especially those features, that can not be taken from data sheets. A procedure was developed to measure and compare light sensitivity, linearity and step response of different cameras. The results show differences in light sensitivity, linearity and step response. The proposed procedure could also be used to measure sensitivity and linearity for quality control.

\section{Acknowledgements}

This work was supported by the Bundesministerium fuir Bildung und Forschung (BMBF) Grant No. 13 N8002 and the Thïringer Ministerium für Wissenschaft, Forschung und Kultur (TMWFK) Grant No. B 308-00018.

\section{References}

[1] R. H. Eikelboom, K. Yogesan, C. J. Barry, et al., "Methods and limits of digital image compression of retinal images for telemedicine", Invest Ophthalmol. Vis. Sci., vol. 41-7, pp. 1916-1924, 2000

[2] P. K. Jensen and E. Scherfig, "Resolution of retinal digital colour images", Acta Ophthalmol. Scand., vol. 77-5, pp. 526-529, 1999
131 I.. J. Bour, L. Koo, F. C. Delori, et al., "Fundus photography for measurement of macular pigment density distribution in children", Invest Ophthalmol. Vis. Sci., vol. 43-5, pp. 1450-1455. $2(X) 2$

141 "Einführung in die CCD-Technologie" (in German) htlp://www.pco.de/. /Knowhow/CCD-Technology.

[5] Hitachi, Technical information, 2002, see e.g. http://www. hituchi-service.net/kp-ml/KP. MI_20timing_20chart.jpg

[6] R. P. Tornow, S. Beuel and E. Zrenner, "Modifying a Rodenstock scanning laser ophthalmoscope for imaging densitometry", Applied Optics, vol. 36-22, pp. 5621-5629, 1997 